\title{
Commentary: Beyond the face: how context modulates emotion processing in frontotemporal dementia subtypes
}

\author{
Liang Chen ${ }^{1,2}$ and Xu Chen ${ }^{1,2 *}$ \\ ${ }^{1}$ Faculty of Psychology, Southwest University, Chongqing, China, ${ }^{2}$ Research Center of Mental Health Education, Southwest \\ University, Chongqing, China
}

Keywords: facial expressions, bodily expressions, emotion recognition, frontotemporal dementia, neuroimaging

\section{A Commentary on}

Beyond the face: how context modulates emotion processing in frontotemporal dementia subtypes

by Kumfor, F., Ibanez, A., Hutchings, R., Hazelton, J. L., Hodges, J. R., and Piguet, O. (2018). Brain 141, 1172-1185. doi: 10.1093/brain/awy002

\section{OPEN ACCESS}

Edited by:

Guido Gainotti,

Catholic University of the Sacred

Heart, Italy

Reviewed by:

Xiangru Zhu,

Henan University, China

Davide Quaranta,

Catholic University of the Sacred

Heart, Italy

*Correspondence:

Xu Chen

chenxu@swu.edu.cn

Received: 04 December 2019

Accepted: 16 July 2020

Published: 20 August 2020

Citation:

Chen L and Chen X (2020)

Commentary: Beyond the face: how context modulates emotion processing in frontotemporal dementia subtypes.

Front. Aging Neurosci. 12:244. doi: 10.3389/fnagi.2020.00244
The first case of language variant of frontotemporal dementia (FTD) was reported by Pick (1892), in which an autopsy revealed focal atrophy of the temporal lobe. Mesulam (1982) reported six patients with a slowly progressing aphasic disorder later called "primary progressive aphasia." The lesion was located at the site of the focal left perisylvian or temporal lobe, but these patients did not have global symptoms of dementia, including additional intellectual and behavioral disturbances. Some separate cases of right frontal or temporal degeneration were subsequently reported, as patients did not recognize their family (i.e., prosopagnosia), nor did they remember spatial topography. A consensus of "frontotemporal lobar degeneration" was put forth by Neary et al. (1998) and Snowden et al. (2002). Recently, the updated relevant diagnostic criteria and classification system with greater sensitivity have also been proposed (Gorno-Tempini et al., 2011; Rascovsky et al., 2011). Evidence from epidemiological studies has shown that FTD is the most common type of primary degenerative dementia, after Alzheimer's disease and dementia with Lewy bodies, accounting for about 20\% of presenile dementia (Ratnavalli et al., 2002; Snowden et al., 2002; Coyle-Gilchrist et al., 2016). Two clinical subtypes of FTD are recognized in the literature. The first clinical subtype of FTD is called behavioral variant frontotemporal dementia (bvFTD), in which the most pronounced brain atrophy is in the mesial and orbitofrontal regions and also includes the temporal lobe (Rosen et al., 2002a, 2005; Peters et al., 2006; Whitwell et al., 2009). In some patients, the alteration of limbic network has been observed (e.g., the insula, striatum, anterior cingulate, and amygdala) (Boccardi et al., 2005; Rascovsky et al., 2011). Their primary motor cortex generally remains intact (Whitwell et al., 2009). The patients with bvFTD have some key clinical characteristics (e.g., disinhibition, apathy, loss of empathy, compulsive behaviors, executive dysfunction) (Rascovsky et al., 2011). The second clinical subtype of FTD is semantic dementia (SD) that presents the defining feature of impairments in naming objects, word comprehension, and other conceptual or semantic knowledge (Hodges et al., 1992; Landin-Romero et al., 2016). The left-lateralized anterior temporal lobe is the primary region of atrophy in SD. In addition, degeneration of the temporal and limbic areas is also reported (Mummery et al., 2000; Yang et al., 2012). Note that about $30 \%$ of cases, however, present with predominantly right-lateralized brain 
atrophy, which is accompanied by various behavioral symptoms (e.g., social disinhibition, emotion recognition, and aggression) and emotional changes (e.g., depression) (Chan et al., 2009; Kumfor et al., 2016).

Social cognitive impairment exists in both bvFTD and SD. In daily life, social cognition typically involves being able to identify others' states correctly, especially their emotional state based on facial expressions. Given these cognitive and neurological abnormalities mentioned above, people with bvFTD and SD have impaired processing of emotions (Kumfor and Piguet, 2012). Patients with both bvFTD and SD show disrupted recognition of basic negative facial expressions (i.e., sad, fearful, disgust, and angry) compared with healthy controls. However, they can recognize happy facial expressions, which involves dissociable brain regions (Kumfor et al., 2013). The recognition of happy faces generally intact in bvFTD, but this pattern in SD relies on the test stimuli used (Kumfor and Piguet, 2012). The identification accuracy of emotional faces in terms of social cognition and emotional assessment significantly relates to gray matter volume in the ventromedial prefrontal cortex in bvFTD (Bertoux et al., 2012). FTD also shows lower performance in tracking dynamic emotional stimuli, which is primarily associated with gray matter loss in the right lateral orbitofrontal cortex (Goodkind et al., 2012). These neurological abnormalities further impair the patient's future thinking, empathy, and theory of mind (Landin-Romero et al., 2016).

However, previous studies have mainly focused on isolated emotional cues (e.g., face, voice), and these stimuli do not encompass the complete range of human emotions (Aviezer et al., 2012b, 2017). Recent studies have demonstrated the importance of context in discriminating between facial expressions. Meeren et al. (2005) first discovered the automatic integration of facial expressions and bodily expressions in an early time window ( $\sim 115 \mathrm{~ms})$, using event-related potentials technique. Moreover, the categorization of facial expressions (e.g., angry, disgust, sad, and fearful) depends on their accompanied bodily expressions (Aviezer et al., 2008). This effect occurs not only for facial expressions that convey approximate values (e.g., angry and disgust) but also for expressions that convey significant differences in values (e.g., win and lose) (Aviezer et al., 2012a). Although deficits in facial emotion recognition in both bvFTD and SD have been thoroughly investigated (Kumfor and Piguet, 2012), it is unclear whether contextual stimuli facilitate or enhance emotion recognition in these patients. Previous research has indirectly investigated the difficulty in understanding contextual information in patients with FTD. For example, bvFTD patients show abnormalities in interpreting sarcastic, but not sincere statements, which is linked to the disproportionate atrophy in the right amygdala, right lateral orbitofrontal cortex, right temporal pole (Kipps et al., 2009), and precuneus (Kumfor et al., 2017). Findings of Kipps et al. (2009) and Kumfor et al. (2017) may be explained as dysfunctions in integrating discordant contextual cues (e.g., voice and face) to judge the true intent of others (Ibanez and Manes, 2012). The social context network model (SCNM) has recently been proposed (Ibanez and Manes, 2012; Baez et al., 2017), which holds that social situations are commonly context-embedded. The integration of target and contextual information is underpinned by a frontal-insular-temporal network: (a) updating context cues to make predictions by the involvement of several frontal areas (i.e., orbitofrontal cortex, lateral prefrontal cortex, and superior orbital sulcus), (b) interaction between internal and external milieus in the insular cortex, and (c) context-target associative learning in the temporal lobe. The SCNM describes the deficits of social cognition (e.g., decision-making, figurative language, facial recognition, empathy, and theory of mind) in bvFTD patients as context impairments. Note that emotional integration and the core atrophic areas in FTD patients feature overlap in their underlying neuroanatomical correlates (e.g., the frontal and temporal areas, limbic network) (de Gelder, 2006; Van den Stock et al., 2014; Hortensius et al., 2016).

These topics were addressed in a recent study published in the journal of Brain; Kumfor and colleagues conducted a series of neuropsychological tests, emotion processing tasks, and a wholebrain structural MRI. First, a pre-study neuropsychological assessment was conducted. Second, all participants completed tasks A-C: recognition of isolated facial expressions (i.e., face only) in task A; recognition of bodily context (i.e., body language only) in task B; contextual effects of body language on the face in task C. Similar to previous studies (e.g., Aviezer et al., 2008), these tasks focused only on the emotions of anger, disgust, fear, and sadness. Categorization accuracy in tasks A and B were calculated, along with contextual influence (i.e., the percentage of times faces were identified as expressing the bodily context emotion) from task C. Finally, high-resolution T1 structural images were acquired. A total of 51 subjects ( 19 with bvFTD, 12 with SD, and 20 controls) participated in all the tests.

Their results revealed the following: first, over-reliance on contextual information appeared in people with bvFTD. Specifically, in recognition of isolated emotions from facial and body language cues (i.e., tasks $\mathrm{A}$ and $\mathrm{B}$ ), people with bvFTD and SD showed similar lower accuracy than did the controls. While categorizing facial expressions in incongruent contexts, those with bvFTD showed worse accuracy and were more likely to be deceived by bodily stimuli than were both people with SD and the controls. Second, there was a similar response pattern in contextual influence between people with $\mathrm{SD}$ and the control group. The perceived similarity between facial expressions and contextual emotion could be a crucial determinant of incongruent face-body pairings in the SD group, as well as in the controls. For instance, an angry face was roughly similar to a disgusted face. Thus, when a disgusted body was presented as context, the face was labeled as angry. Third, the right parahippocampal/amygdala and left precentral gyrus were associated with categorization accuracy and contextual influence in people with bvFTD and those with SD. Generally, the lower integrity between these two regions related to a higher degree of contextual influence. Moreover, lower integrity of the bilateral temporal fusiform cortex and orbitofrontal cortex was also observed in bvFTD. Although the left precentral gyrus is not typically reported to be involved in facial expression processing, its increased activation has been found when viewing bodily images (Kourtzi and Kanwisher, 2000), gestures, actions, and implied motion (Saggar et al., 2014; Kolesar et al., 2017). 
Kumfor et al. (2018)'s findings offer a unique opportunity to demonstrate the relative contributions of the right parahippocampal/amygdala and left precentral gyrus for the interaction of contextual information and emotion processing. While a recent study with a group that underwent unilateral anterior temporal lobe resections, including the amygdala, has suggested that the amygdala and anterior temporal lobe are not necessary for recognition of dynamic bodily expressions (Van de Vliet et al., 2018), Kumfor et al. (2018) explored a more real-life situation, that is, emotion recognition with contextual information. The greater contextual influence in bvFTD patients reflects difficulty in integrating and modulating emotional contextual information when recognizing the target, which might be akin to the 'environmental dependency syndrome' described in frontal lesion patients (Lhermitte, 1983, 1986; Lhermitte et al., 1986). Specifically, individuals could have inappropriate behaviors that are induced by external clues or cues, regardless of the intended goals and emotional consequences. The findings of Kumfor et al. (2018) also supports the SCNM. The frontal lobe lesions might result in the activation of automatic reliance on the emotional context when recognizing the target (e.g., facial expression) by the external clues (e.g., bodily expression) in bvFTD patients. In the SCNM, frontal areas are in charge of updating context cues to make predictions. Kumfor et al. (2018)'s results suggest that contextual influence is sensitive to social cognition impairment and is specific to bvFTD patients. However, unlike bvFTD patients, SD patients' performance in the task $\mathrm{C}$ is modulated by context, indicating a degree of contextual integration. The SCNM proposes that temporal lobe is responsible for context-target associative learning, via basic related process with the perirhinal cortex, hippocampus, and amygdala. Kumfor et al. (2018) also revealed that abnormal contextual influence was associated with lower integrity of the right parahippocampal gyrus/amygdala and left precentral gyrus. Therefore, these behavioral and neuroimaging findings are in accordance with the SCNM.

Moreover, we suggest some clinical implications here. Early emotional blunting, which refers to an inappropriate emotional shallowness with unconcern and a loss of emotional warmth, empathy, sympathy, and an indifference to others, is one of the core diagnostic features in people with FTD (Gorno-Tempini et al., 2011; Rascovsky et al., 2011). The difficulty in facial expressions recognition has also been thoroughly investigated in both people with bvFTD and SD (Lavenu et al., 1999; Rosen et al., 2002b; Kumfor and Piguet, 2012; Kumfor et al., 2013). The different response patterns (i.e., over-reliance on contextual information observed in bvFTD, rather than SD) might provide a relative reference for diagnosing subtypes of global FTD and enrich our understanding of social cognition impairment in these groups. Future research could also build on the contextual emotion recognition task, use more technical measures, and develop multimodal biomarkers for diagnosis of FTD including, but not limited to, whole-brain structural MRI and behavioral performance. Facial expressiveness and physiological responses (e.g., skin conductance level, SCL) in FTD when processing emotional stimuli are worth considering. For instance, in Kumfor et al. (2019), bvFTD patients, SD patients, and healthy controls were required to passively view emotional video clips, and surface facial electromyography (EMG) and SCL were recorded. SD patients showed increased zygomaticus reactions to neutral and positive videos, but bvFTD had the same facial EMG responses in all conditions. Both FTD patient groups did not show greater SCL when viewing emotional videos, unlike controls. Patients with bvFTD also have difficulties to imitate facial expressions (Gola et al., 2017). Note that simultaneous heart rate and pupillometric responses are meaningful as well, because different subtypes of FTD may have slight differences in these two indicators when processing complex socio-emotional stimuli. For example, Marshall et al. (2019) performed a task fMRI with simultaneous heart rate and pupillometric recordings, during which four groups (bvFTD; semantic variant primary progressive aphasia, svPPA; non-fluent variant primary progressive aphasia, nvPPA; healthy controls) were required to passively view dynamic universal emotional facial expressions. They found that, compared to healthy controls, all syndromes showed significant impairments in cardiac reactivity, and only individuals with nvPPA had impaired pupillary reactivity. They also revealed some syndrome-specific activations to predict facial emotion recognition accuracy (e.g., anterior insula and caudate for bvFTD, anterior temporal cortex for svPPA). Therefore, the recent studies provide grounds for future researchers to incorporate technical measures to systematically develop the multimodal biomarkers of FTD in contextual emotion recognition. In addition, future interventions might reduce over-reliance on contextual information in people with bvFTD through priming or subliminal presentation. Furthermore, congruent contextual information could be provided during treatment to improve the patients' abilities regarding emotion recognition in both those with bvFTD and SD. These hypotheses will be examined in the future.

In summary, the authors (Kumfor et al., 2018) provide theoretically and clinically significant evidence of how contextual information modulates emotion recognition in the FTD subtypes. Future research is warranted to expand the sample size of patients with SD to improve statistical power, especially for right-lateralized SD patients. Moreover, it is needed to recruit a large sample of both healthy adults and neurological patients to validate the three tasks into a clinically sensitive test of contextual emotion recognition, like the classical TASIT (Henry et al., 2016). Note that some details of these three tasks should be improved. For example, the task A should display one face, rather than an array of seven faces, and use labeling paradigm, which may be comparable with tasks $\mathrm{B}$ and $\mathrm{C}$ for task demand; more basic or complex emotions (e.g., happy, pride) could be included to expand our understanding of social cognition in neurological disorders. Finally, other contextual cues from multiple modalities should be explored, such as scene and voice. Patients with bvFTD are also poor at classifying laughter, retching, crying (Keane et al., 2002), and emotional music (Hsieh et al., 2011, 2012; Fittipaldi et al., 2019). Emotional integration and conflict in healthy people have been widely investigated (Schirmer and Adolphs, 2017), but that in the FTD group is unclear. Findings of Kumfor et al. (2018) are likely to inspire more insights and therapeutic interventions regarding social cognition impairment. 


\section{AUTHOR CONTRIBUTIONS}

All authors listed have made a substantial, direct and intellectual contribution to the work, and approved it for publication.

\section{REFERENCES}

Aviezer, H., Ensenberg, N., and Hassin, R. R. (2017). The inherently contextualized nature of facial emotion perception. Curr. Opin. Psychol. 17, 47-54. doi: 10.1016/j.copsyc.2017.06.006

Aviezer, H., Hassin, R. R., Ryan, J., Grady, C., Susskind, J., Anderson, A., et al. (2008). Angry, disgusted, or afraid? Studies on the malleability of emotion perception. Psychol. Sci. 19, 724-732. doi: 10.1111/j.1467-9280.2008.02148.x

Aviezer, H., Trope, Y., and Todorov, A. (2012a). Body cues, not facial expressions, discriminate between intense positive and negative emotions. Science 338, 1225-1229. doi: 10.1126/science. 1224313

Aviezer, H., Trope, Y., and Todorov, A. (2012b). Holistic person processing: faces with bodies tell the whole story. J. Pers. Soc. Psychol. 103, 20-37. doi: 10.1037/a0027411

Baez, S., Garcia, A. M., and Ibanez, A. (2017). The social context network model in psychiatric and neurological diseases. Curr. Top. Behav. Neurosci. 30, 379-396. doi: 10.1007/7854_2016_443

Bertoux, M., Volle, E., Funkiewiez, A., de Souza, L. C., Leclercq, D., and Dubois, B. (2012). Social cognition and emotional assessment (sea) is a marker of medial and orbital frontal functions: a voxel-based morphometry study in behavioral variant of frontotemporal degeneration. J. Int. Neuropsychol. Soc. 18, 972-985. doi: $10.1017 /$ S1355617712001300

Boccardi, M., Sabattoli, F., Laakso, M. P., Testa, C., Rossi, R., Beltramello, A., et al. (2005). Frontotemporal dementia as a neural system disease. Neurobiol. Aging 26, 37-44. doi: 10.1016/j.neurobiolaging.2004.02.019

Chan, D., Anderson, V., Pijnenburg, Y., Whitwell, J., Barnes, J., Scahill, R., et al. (2009). The clinical profile of right temporal lobe atrophy. Brain 132, 1287-1298. doi: 10.1093/brain/awp037

Coyle-Gilchrist, I. T., Dick, K. M., Patterson, K., Rodríquez, P. V., Wehmann, E., Wilcox, A., et al. (2016). Prevalence, characteristics, and survival of frontotemporal lobar degeneration syndromes. Neurology 86, 1736-1743. doi: 10.1212/WNL.0000000000002638

de Gelder, B. (2006). Towards the neurobiology of emotional body language. Nat. Rev. Neurosci. 7, 242-249. doi: 10.1038/nrn1872

Fittipaldi, S., Ibanez, A., Baez, S., Manes, F., Sedeno, L., and García, A. M. (2019). More than words: social cognition across variants of primary progressive aphasia. Neurosci. Biobehav. Rev. 100, 263-284. doi: 10.1016/j.neubiorev.2019.02.020

Gola, K. A., Shany-Ur, T., Pressman, P., Sulman, I., Galeana, E., Paulsen, H., et al. (2017). A neural network underlying intentional emotional facial expression in neurodegenerative disease. Neuroimage Clin. 14, 672-678. doi: 10.1016/j.nicl.2017.01.016

Goodkind, M. S., Sollberger, M., Gyurak, A., Rosen, H. J., Rankin, K. P., Miller, B., et al. (2012). Tracking emotional valence: the role of the orbitofrontal cortex. Hum. Brain Mapp. 33, 753-762. doi: 10.1002/hbm.21251

Gorno-Tempini, M. L., Hillis, A. E., Weintraub, S., Kertesz, A., Mendez, M., Cappa, S. F., et al. (2011). Classification of primary progressive aphasia and its variants. Neurology 76, 1006-1014. doi: 10.1212/WNL.0b013e31821103e6

Henry, J. D., Von Hippel, W., Molenberghs, P., Lee, T., and Sachdev, P. S. (2016). Clinical assessment of social cognitive function in neurological disorders. Nat. Rev. Neurol. 12, 28-39. doi: 10.1038/nrneurol.2015.229

Hodges, J. R., Patterson, K., Oxbury, S., and Funnell, E. (1992). Semantic dementia - progressive fluent aphasia with temporal-lobe atrophy. Brain 115, 1783-1806. doi: 10.1093/brain/115.6.1783

Hortensius, R., Terburg, D., Morgan, B., Stein, D. J., van Honk, J., and de Gelder, B. (2016). The role of the basolateral amygdala in the perception of faces in natural contexts. Philos. Trans. R. Soc. B 371:20150376. doi: 10.1098/rstb.2015.0376

Hsieh, S., Hornberger, M., Piguet, O., and Hodges, J. R. (2011). Neural basis of music knowledge: evidence from the dementias. Brain 134, 2523-2534. doi: 10.1093/brain/awr190

\section{FUNDING}

This work was supported by the National Natural Science Foundation of China (Grant No. 31771232).

Hsieh, S., Hornberger, M., Piguet, O., and Hodges, J. R. (2012). Brain correlates of musical and facial emotion recognition: evidence from the dementias. Neuropsychologia 50, 1814-1822. doi: 10.1016/j.neuropsychologia.2012.04.006

Ibanez, A., and Manes, F. (2012). Contextual social cognition and the behavioral variant of frontotemporal dementia. Neurology 78, 1354-1362. doi: 10.1212/WNL.0b013e3182518375

Keane, J., Calder, A. J., Hodges, J. R., and Young, A. W. (2002). Face and emotion processing in frontal variant frontotemporal dementia. Neuropsychologia 40, 655-665. doi: 10.1016/S0028-3932(01)00156-7

Kipps, C. M., Nestor, P. J., Acosta-Cabronero, J., Arnold, R., and Hodges, J. R. (2009). Understanding social dysfunction in the behavioural variant of frontotemporal dementia: the role of emotion and sarcasm processing. Brain 132, 592-603. doi: 10.1093/brain/awn314

Kolesar, T. A., Kornelsen, J., and Smith, S. D. (2017). Separating neural activity associated with emotion and implied motion: an fMRI study. Emotion 17, 131-140. doi: 10.1037/emo0000209

Kourtzi, Z., and Kanwisher, N. (2000). Activation in human $\mathrm{mt} / \mathrm{mst}$ by static images with implied motion. J. Cogn. Neurosci. 12, 48-55. doi: 10.1162/08989290051137594

Kumfor, F., Hazelton, J. L., Rushby, J. A., Hodges, J. R., and Piguet, O. (2019). Facial expressiveness and physiological arousal in frontotemporal dementia: Phenotypic clinical profiles and neural correlates. Cogn. Affect. Behav. Neurosci. 19, 197-210. doi: 10.3758/s13415-018-00658-Z

Kumfor, F., Honan, C., McDonald, S., Hazelton, J. L., Hodges, J. R., and Piguet, O. (2017). Assessing the "social brain" in dementia: applying TASIT-S. Cortex 93, 166-177. doi: 10.1016/j.cortex.2017.05.022

Kumfor, F., Ibanez, A., Hutchings, R., Hazelton, J. L., Hodges, J. R., and Piguet, O. (2018). Beyond the face: how context modulates emotion processing in frontotemporal dementia subtypes. Brain 141, 1172-1185. doi: 10.1093/brain/awy002

Kumfor, F., Irish, M., Hodges, J. R., and Piguet, O. (2013). Discrete neural correlates for the recognition of negative emotions: insights from frontotemporal dementia. PLoS ONE 8:e67457. doi: 10.1371/journal.pone.0067457

Kumfor, F., Landin-Romero, R., Devenney, E., Hutchings, R., Grasso, R., Hodges, J. R., et al. (2016). On the right side? A longitudinal study of left- versus rightlateralized semantic dementia. Brain 139, 986-998. doi: 10.1093/brain/awv387

Kumfor, F., and Piguet, O. (2012). Disturbance of emotion processing in frontotemporal dementia: a synthesis of cognitive and neuroimaging findings. Neuropsychol. Rev. 22, 280-297. doi: 10.1007/s11065-0129201-6

Landin-Romero, R., Tan, R., Hodges, J. R., and Kumfor, F. (2016). An update on semantic dementia: genetics, imaging, and pathology. Alzheimers Res. Ther. 8, 52. doi: 10.1186/s13195-016-0219-5

Lavenu, I., Pasquier, E., Lebert, F., Petit, H., and Van der Linden, M. (1999). Perception of emotion in frontotemporal dementia and alzheimer disease. Alzheimer Dis. Assoc. Disord. 13, 96-101. doi: 10.1097/00002093-199904000-00007

Lhermitte, F. (1983). 'Utilization behaviour'and its relation to lesions of the frontal lobes. Brain 106, 237-255. doi: 10.1093/brain/106.2.237

Lhermitte, F. (1986). Human autonomy and the frontal lobes. Part II: patient behavior in complex and social situations: the 'environmental dependency syndrome'. Ann. Neurol. 19, 335-343. doi: 10.1002/ana.410190405

Lhermitte, F., Pillon, B., and Serdaru, M. (1986). Human autonomy and the frontal lobes. Part I: imitation and utilization behavior: a neuropsychological study of 75 patients. Ann. Neurol. 19, 326-334. doi: 10.1002/ana.4101 90404

Marshall, C. R., Hardy, C. J., Russell, L. L., Bond, R. L., Sivasathiaseelan, H., Greaves, C., et al. (2019). The functional neuroanatomy of emotion processing in frontotemporal dementias. Brain 142, 2873-2887. doi: 10.1093/brain/awz204 
Meeren, H. K., van Heijnsbergen, C., and de Gelder, B. (2005). Rapid perceptual integration of facial expression and emotional body language. Proc. Natl. Acad. Sci. U.S.A. 102, 16518-16523. doi: 10.1073/pnas.0507650102

Mesulam, M. M. (1982). Slowly progressive aphasia without generalized dementia. Ann. Neurol. 11, 592-598. doi: 10.1002/ana.410110607

Mummery, C. J., Patterson, K., Price, C. J., Ashburner, J., Frackowiak, R. S. J., and Hodges, J. R. (2000). A voxel-based morphometry study of semantic dementia: Relationship between temporal lobe atrophy and semantic memory. Ann. Neurol. 47, 36-45. doi: 10.1002/1531-8249(200001)47:13.0.CO;2-L

Neary, D., Snowden, J. S., Gustafson, L., Passant, U., Stuss, D., Black, S., et al. (1998). Frontotemporal lobar degeneration - a consensus on clinical diagnostic criteria. Neurology 51, 1546-1554. doi: 10.1212/Wnl.51.6.1546

Peters, F., Perani, D., Herholz, K., Holthoff, V., Beuthien-Baumann, B., Sorbi, S., et al. (2006). Orbitofrontal dysfunction related to both apathy and disinhibition in frontotemporal dementia. Dement. Geriatr. Cogn. Disord. 21, 373-379. doi: $10.1159 / 000091898$

Pick, A. (1892). Uber die beziehungen der senilen hirnatrophie zur aphasie. Prager Med. Wochenschr. 17, 165-167.

Rascovsky, K., Hodges, J. R., Knopman, D., Mendez, M. F., Kramer, J. H., Neuhaus, J., et al. (2011). Sensitivity of revised diagnostic criteria for the behavioural variant of frontotemporal dementia. Brain 134, 2456-2477. doi: 10.1093/brain/awr179

Ratnavalli, E., Brayne, C., Dawson, K., and Hodges, J. R. (2002). The prevalence of frontotemporal dementia. Neurology 58, 1615-1621. doi: 10.1212/WNL.58.11.1615

Rosen, H. J., Allison, S. C., Schauer, G. F., Gorno-Tempini, M. L., Weiner, M. W., and Miller, B. L. (2005). Neuroanatomical correlates of behavioural disorders in dementia. Brain 128, 2612-2625. doi: 10.1093/brain/awh628

Rosen, H. J., Gorno-Tempini, M. L., Goldman, W. P., Perry, R. J., Schuff, N., Weiner, M., et al. (2002a). Patterns of brain atrophy in frontotemporal dementia and semantic dementia. Neurology 58, 198-208. doi: 10.1212/Wnl.58.2.198

Rosen, H. J., Perry, R. J., Murphy, J., Kramer, J. H., Mychack, P., Schuff, N., et al. (2002b). Emotion comprehension in the temporal variant of frontotemporal dementia. Brain 125, 2286-2295. doi: 10.1093/brain/awf225

Saggar, M., Shelly, E. W., Lepage, J. F., Hoeft, F., and Reiss, A. L. (2014). Revealing the neural networks associated with processing of natural social interaction and the related effects of actor-orientation and face-visibility. Neuroimage 84, 648-656. doi: 10.1016/j.neuroimage.2013.09.046

Schirmer, A., and Adolphs, R. (2017). Emotion perception from face, voice, and touch: comparisons and convergence. Trends Cogn. Sci. 21, 216-228. doi: 10.1016/j.tics.2017.01.001

Snowden, J. S., Neary, D., and Mann, D. M. A. (2002). Frontotemporal dementia. Br. J. Psychiatry 180, 140-143. doi: 10.1192/bjp.180.2.140

Van de Vliet, L., Jastorff, J., Huang, Y. A., Van Paesschen, W., Vandenbulcke, M., and Van den Stock, J. (2018). Anterior temporal lobectomy impairs neural classification of body emotions in right superior temporal sulcus and reduces emotional enhancement in distributed brain areas without affecting behavioral classification. J. Neurosci. 38, 9263-9274. doi: 10.1523/JNEUROSCI.0634-1 8.2018

Van den Stock, J., Vandenbulcke, M., Sinke, C. B. A., Goebel, R., and de Gelder, B. (2014). How affective information from faces and scenes interacts in the brain. Soc. Cogn. Affect. Neurosci. 9, 1481-1488. doi: 10.1093/scan/ nst 138

Whitwell, J. L., Przybelski, S. A., Weigand, S. D., Ivnik, R. J., Vemuri, P., Gunter, J. L., et al. (2009). Distinct anatomical subtypes of the behavioural variant of frontotemporal dementia: a cluster analysis study. Brain 132, 2932-2946. doi: 10.1093/brain/awp232

Yang, J., Pan, P. L., Song, W., and Shang, H. F. (2012). Quantitative meta-analysis of gray matter abnormalities in semantic dementia. J. Alzheimers Dis. 31, 827-833. doi: 10.3233/Jad-2012-120736

Conflict of Interest: The authors declare that the research was conducted in the absence of any commercial or financial relationships that could be construed as a potential conflict of interest.

Copyright (c) 2020 Chen and Chen. This is an open-access article distributed under the terms of the Creative Commons Attribution License (CC BY). The use, distribution or reproduction in other forums is permitted, provided the original author(s) and the copyright owner(s) are credited and that the original publication in this journal is cited, in accordance with accepted academic practice. No use, distribution or reproduction is permitted which does not comply with these terms. 